\title{
Symmetry-Dependent Spin Transport Properties and Spin-Filter Effects in Zigzag-Edged Germanene Nanoribbons
}

\author{
Can Cao, ${ }^{1,2}$ Mengqiu Long, ${ }^{1}$ and Xiancheng $\mathrm{Mao}^{2}$ \\ ${ }^{1}$ School of Physics and Electronics, Central South University, Changsha 410083, China \\ ${ }^{2}$ School of Geosciences and Info-Physics, Central South University, Changsha 410083, China \\ Correspondence should be addressed to Mengqiu Long; mqlong@csu.edu.cn
}

Received 26 March 2015; Revised 2 July 2015; Accepted 5 July 2015

Academic Editor: Christian Brosseau

Copyright (c) 2015 Can Cao et al. This is an open access article distributed under the Creative Commons Attribution License, which permits unrestricted use, distribution, and reproduction in any medium, provided the original work is properly cited.

\begin{abstract}
We performed the first-principles calculations to investigate the spin-dependent electronic transport properties of zigzag-edged germanium nanoribbons (ZGeNRs). We choose of ZGeNRs with odd and even widths of 5 and 6 , and the symmetry-dependent transport properties have been found, although the $\sigma$ mirror plane is absent in ZGeNRs. Furthermore, even- $N$ and odd- $N$ ZGeNRs have very different current-voltage relationships. We find that the even 6-ZGeNR shows a dual spin-filter effect in antiparallel (AP) magnetism configuration, but the odd 5-ZGeNR behaves as conventional conductors with linear current-voltage dependence. It is found that when the two electrodes are in parallel configuration, the 6-ZGeNR system is in a low resistance state, while it can switch to a much higher resistance state when the electrodes are in AP configuration, and the magnetoresistance of $270 \%$ can be observed.
\end{abstract}

\section{Introduction}

Graphene and graphene nanoribbons (GNRs) have attracted broad academic and industrial interests owing to their amazing properties $[1,2]$ and potential applications in nanodevices [3-5]. In particular, their applications in spintronics field [6-8] offer the most promising solutions for future high operating speed and energy-saving electronic devices. For instance, the zigzag graphene nanoribbon (ZGNR) is an antiferromagnetic insulator with considerable magnetic moment located at edge sites, which give rise to many unusual properties, such as half metallic [9], magnetoelectric effect $[10,11]$, and spin-filter effect $[11,12]$.

These interesting behaviors of graphene motivate the further exploration of honeycomb lattice with higher group IV elements. Walking down the periodic table from carbon to silicon $(\mathrm{Si})$ to germanium $(\mathrm{Ge})$ enhances the $d$-orbital participation in their electronic behavior and thus develops strong electronic correlation. And there are many interesting magnetic and charge excitations behaviors that would be introduced. Theory predicts free standing silicene or germanene sheets and ribbons to be stable in a low puckered configuration [13-16]. Different from graphene, in silicene or germanene, two adjacent atoms belonging to the same sublattice are not in the same plane owing to the buckled structure of silicene or germanene. Thus, the buckled structural feature would give rise to tunable spin-valley coupled band structures, which accounts for many exotic transport [17, 18] and superconducting phenomena [19] and makes silicene and germanene good candidates for the spintronics [20-23]. The first-principle calculations show that the intrinsic carrier mobility of germanene sheet can even reach $\sim 6 \times 10^{5} \mathrm{~cm}^{2} \mathrm{~V}^{-1} \mathrm{~s}^{-1}$, which is larger than that of graphene [24]. In particular, compared with $\mathrm{Si}$, Ge has longer spin-diffusion length and the relative strong spin-orbit coupling would be helpful to the spin injection and the manipulation of spin $[25,26]$. Thus, the developments of multifunctional germanene-based spintronics components and the corresponding nanodevices design are more worth studying thoroughly. A feasible approach to realize germanene-based spintronics is to construct the device junctions of germanium nanoribbons (ZGeNRs) and then systematically study the transport properties as fundamental and crucial components of germanene-based circuits. Recently, intriguing transport phenomena have been found in zigzag graphene nanoribbons (ZGNRs) [27], zigzag 


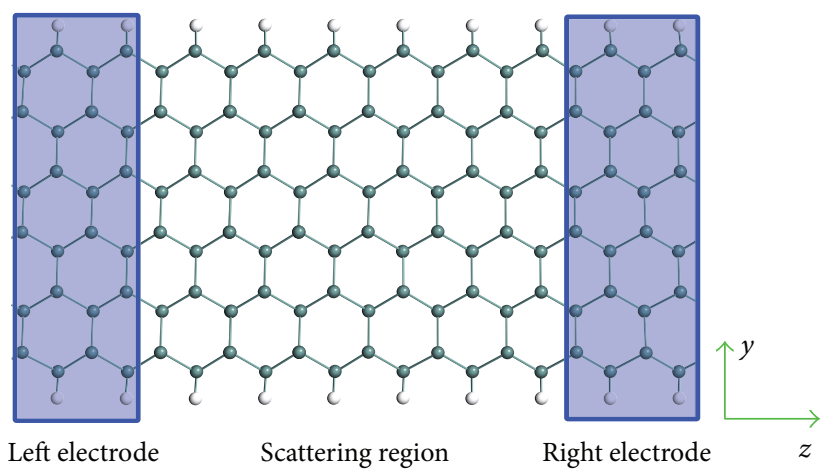

(a)

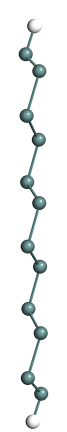

(b)

FIGURE 1: Two-probe system of 6-ZGeNR device: (a) top view and (b) cross section view. White and green balls represent $\mathrm{H}$ and Ge atoms, respectively. The green arrow corresponds to $y$ - and $z$-axis.

$\alpha$-graphyne nanoribbons [28], and silicene nanoribbons (ZSiNRs) with buckled structure [21], where symmetric and asymmetric zigzag ribbons are found to have completely different $I-V$ characteristics despite the similarity of their band structure. More interestingly, bipolar spin diode behavior was observed in symmetry devices. Spin polarization and direction of the current through the device can be controlled through either the voltage or magnetic configuration of the electrodes. Such freedom in controlling the spin-polarized current is useful in design of spin devices such as rectification and amplification for spintronics. However, until now, no one is working on the spin transport property of symmetric and asymmetric ZGeNRs. Developments of multifunctional germanium-based spintronics devices that offer effective manipulation of spin-polarized current are an important supplement of exploration of spintronics.

So, in this work, we investigate the spin-dependent transport properties of ZGeNRs by first-principles calculations. Our results show that although the $\sigma$ plane is absent in ZGeNRs due to the low puckered configuration, the ZGeNRs still exhibit symmetry-dependent transport properties. And the magnetoresistance effect is also observed in ZGeNRs. Our works suggest a route to manipulate spin-polarized current and to design the germanene-based spintronic devices.

\section{Models and Theoretical Method}

ZGeNRs can be classified by the number of Ge atoms along the width of the ribbon. We choose ZGeNRs with odd and even widths of 5 and 6 , which are denoted here as 5-ZGeNR and $6-Z G e N R$, respectively. The geometric structures from top view and cross section view of 6-ZGeNR are shown in Figures $1(\mathrm{a})$ and $1(\mathrm{~b})$, respectively. The system is divided into three regions: left electrode, right electrode, and the central scattering region. Each electrode is described by a supercell with two repeated ZGeNR unit cells along transport direction, and the scattering region is ZGeNR with length of 6 unit cells. An external magnetic field controlling the magnetization of the left (right) ZGeNR electrode can be set to be 1 or -1 , corresponding to magnetization along the $+x$ or $-x$ direction. In this paper, we use $L_{M}$ and $R_{M}$ to represent the magnetization of the left and right electrodes; that is, $\left(L_{M}, R_{M}\right)=(1,1)$ shows that the left and right electrodes are parallel $(\mathrm{P})$ in spin orientation, while $\left(L_{M}, R_{M}\right)=(1,-1)$ shows that the left and right electrodes are antiparallel (AP) in spin orientation. The geometric optimization and spinresolved electronic transport properties are calculated by a developed $a b$ initio software package Atomistix ToolKit [29, 30], which is based on the spin-polarized density-functional theory combined with the nonequilibrium Green functions. In the calculation, the Troullier-Martins norm-conserving pseudopotential, the local spin density approximation (LSDA) for exchange-correlation potential, and $k$-point grid $1 \times 1 \times 100$ are used. The real space grid techniques are used with the energy cutoff of $150 \mathrm{Ry}$ as a required cutoff energy in numerical integrations and the solution of Poisson equation using fast Fourier transform (FFT). The geometry optimization is performed using quasi-Newton method until the absolute value of force acting on each atom is $<0.05 \mathrm{eV} / \AA$. The wave functions of $\mathrm{Ge}$ and $\mathrm{H}$ atoms are expanded by single-zeta polarized (SZP) basis set. The spin-resolved current is calculated using the Landauer formula:

$$
\begin{aligned}
I_{\sigma} & \left(V_{b}\right) \\
\quad= & \frac{e}{\hbar} \int_{\mu_{L}\left(V_{b}\right)}^{\mu_{R}\left(V_{b}\right)} T_{\sigma}\left(E, V_{\sigma}\right)\left[f_{L}\left(E, V_{\sigma}\right)-f_{R}\left(E, V_{\sigma}\right)\right] d E,
\end{aligned}
$$

where $\sigma=\uparrow$ (spin up) and $\downarrow$ (spin down), $\mu_{L / R}$ is the electrochemical potential of the left/right electrode, and the difference of them is $\mu_{L}-\mu_{R}=e V_{b}$, and $T_{\sigma}\left(E, V_{\sigma}\right)$ is the spin-resolved transmission defined as

$$
T_{\sigma}\left(E, V_{b}\right)=\operatorname{Tr}\left[\Gamma_{L}\left(E, V_{b}\right) G^{R}(E) \Gamma_{R}\left(E, V_{b}\right) G^{A}(E)\right],
$$

where $G^{R(A)}$ is the retarded (advanced) Green functions of the central region and $\Gamma_{L(R)}$ is the contact broadening functions.

\section{Results and Discussions}

The spin configuration is crucial for the spin-filter effect, and many researchers have found that ZGNR, Z $\alpha$ GNRs, and ZSiNR can show perfect spin-filter effect with AP configuration $[12,21,27,28]$. So, in here, as shown in 


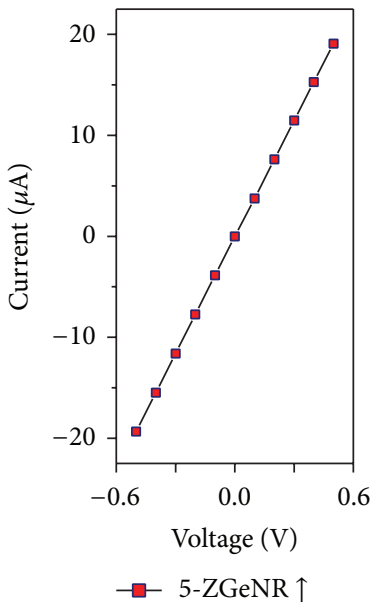

(a)

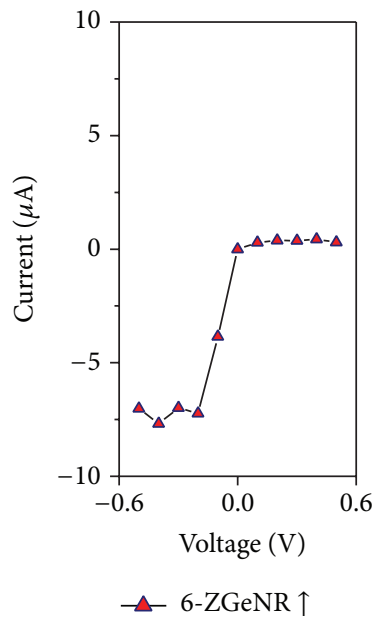

(e)

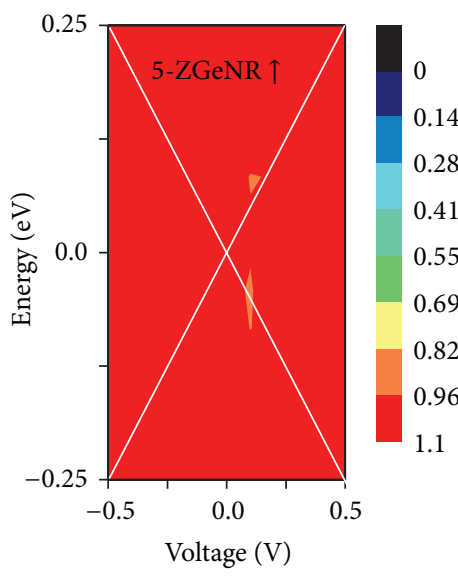

(b)

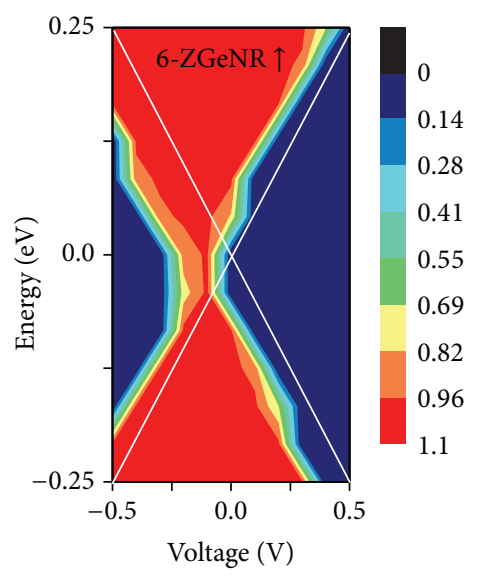

(f)

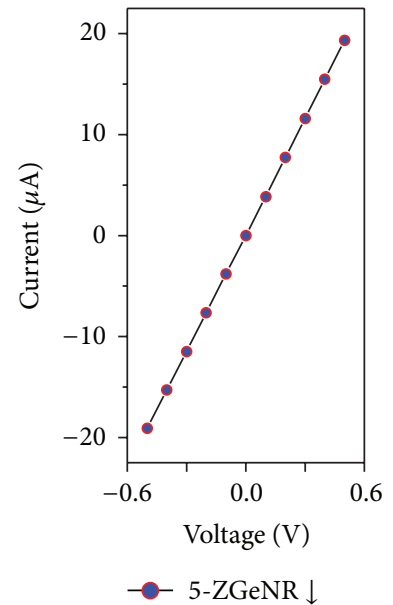

(c)

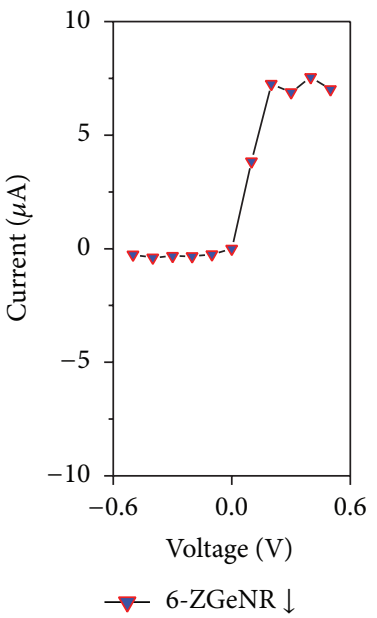

(g)

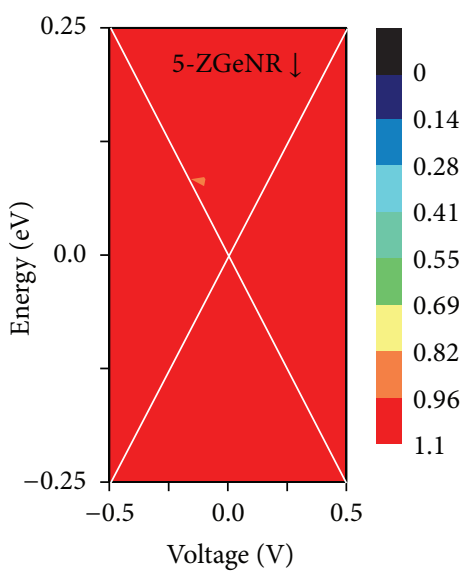

(d)

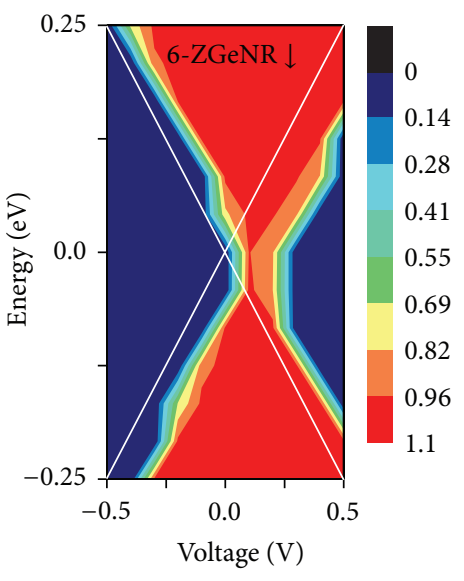

(h)

FIGURE 2: Spin-dependent $I-V$ curves and transmission spectra as a function of electron energy $E$ and bias $V_{b}$. (a, b) Spin-up state of 5-ZGeNR; (c, d) Spin-down state of 5-ZGeNR; (e, f) Spin-up state of 6-ZGeNR; (g, h) Spin-down state of 6-ZGeNR. The region between the solid lines is referred to as bias window, which sets boundaries for transmission that contributes to the current at a given bias voltage. The Fermi level is set to zero.

Figure 2, we consider firstly that the current-voltage $(I-V)$ curves come with the spin-dependent transmission spectrum as a function of the electron energy and bias of 5-ZGeNR and 6-ZGeNR under the AP magnetic configuration. From the Landauer formula (1), we know that the spin-resolved current through central scattering region is decided by the transmission within the considered bias window. Therefore, the properties of $I-V$ curves can be understood based on the transmission spectrum. In Figure 2(b), for spin-up state of 5ZGeNR, the area within the bias window increases linearly under the whole bias window; thus a linear $I-V$ curve can be observed in Figure 2(a). The spin-down state of 5-ZGeNR presents a similar property, as shown in Figures 2(c) and 2(d). Interestingly, as shown in Figures $2(\mathrm{e})-2(\mathrm{~h})$, the $I-V$ curves of 6-ZGeNR can be modulated by the spin orientation of the electrodes and the direction of external $V_{\text {bias }}$. It is clearly seen in Figure 2(f) that the spin-up transmission within the bias window is always zero in the positive bias range, which leads to full suppression of spin-up current $\left(I_{\text {up }}\right)$ under the positive bias as shown in Figure 2(e). Nevertheless, in the negative bias range, the variation of the current with the bias voltage goes through two different stages. When bias is less than $0.20 \mathrm{~V}$, as shown in Figure 2(e), there are more and more transmission coefficients entering into the bias window with the increase of bias voltage, leading to a quick increase of current. When the bias voltage is above $0.2 \mathrm{~V}$, the rate of the increase of transmission in the bias window is slower owing to the presence of regions with smaller transmission coefficient, which lower down the increase of the current as shown in Figure 2(e). The spin-down state of the AF configuration shows a completely opposite property. That is to say, in the positive bias range, only the spin-down current $\left(I_{\mathrm{dn}}\right)$ is allowed to pass through the central scattering region, while in the negative bias range, only the spin-up current is possible. A device with such property is referred to as a bipolar spin diode [6] or a dual spin filter $[9,12]$ due to the unidirectional nature of the spindependent current, which is different from the traditional bipolar devices based on the polarization of the electron or 


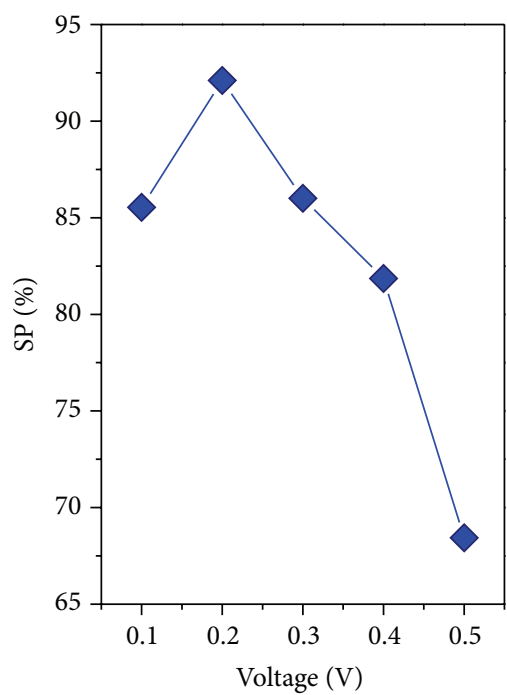

(a)

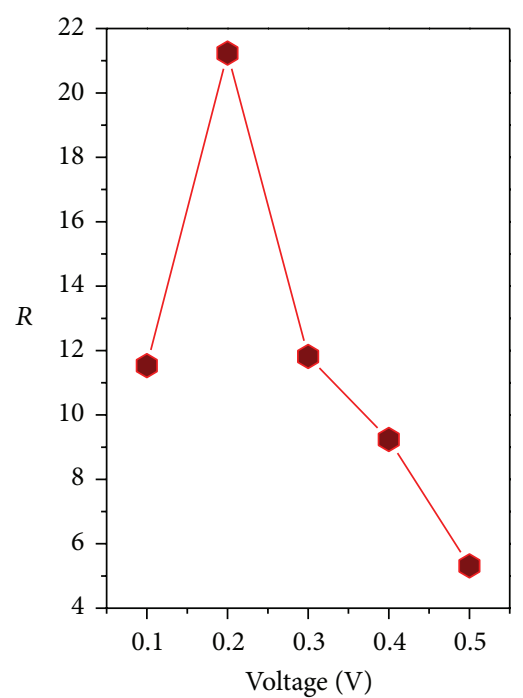

(b)

FIGURE 3: (a) Spin polarization (SP) dependent on the applied bias. (b) Rectifying ratio ( $R$ ) of spin-up current changes with the applied bias.

hole carriers. In contrast, the current for 5-ZGeNR with the AP configuration is almost independent of spin and shows a linear metallic behavior. This interesting phenomenon is very similar to those of $[21,27,28]$, which is possible because of a symmetry selection rule that was first proposed by Li et al. [27]. And, for symmetry one, the flexible control over spin current makes it possible to use the two-terminal device as a basic component for building multifunctional spintronic devices. Compared with other $2 \mathrm{D}$ materials nanoribbons such as ZGNR, Z $\alpha$ GNRs, and ZSiNR spin devices, ZGeNRbased spin devices possess several unique features besides the common point that the spin polarization of the current can be simply tuned by switching either the magnetization of the electrodes or the $V_{\text {bias }}$ direction. First, the intrinsic carrier mobility of germanene is much larger than that of graphene, making it a good candidate of ultrafast devices. Second, germanene is a much better spin transport medium because the spin-diffusion length in germanene is much longer than that in graphene and silicene.

The above results also illuminate high spin polarization and rectifying ratio $(R)$ for $6-\mathrm{ZGeNR}$ in the AP configuration. As shown in Figures 3(a) and 3(b), we calculate the corresponding spin polarization $(\mathrm{SP})$ and rectifying ratio $(R)$, defined by the formulas $\mathrm{SP}=\left(I_{\mathrm{up}}-I_{\mathrm{dn}}\right) /\left(I_{\mathrm{up}}+I_{\mathrm{dn}}\right)$ and $R=I(-V) / I(V)$, respectively. And we can clearly see that the maximum values of SP can reach $92 \%$, and the maximum of rectifying ratio is 22 for the spin-up current of 6-ZGeNR.

These interesting spin transport properties can be understood from the transmission spectra and band structures of the left and right electrodes for the 5-ZGeNR and 6-ZGeNR at zero bias, as shown in Figure 4. From the band structures panels, we can find that the bands of spin-up and spin-down states around FL are split in each system. And also, owing to the different spin orientation, we can find that the subbands of spin-up and spin-down states are opposite on the left and right electrodes. In the central panel of Figure 4(a), we can see the broad transmission platforms for both spin-up and spin-down states in the $5-\mathrm{ZGeNR}$ at zero bias. As the bias increases, the transmission probability remains to be one $G_{0}$ ( $G_{0}=e^{2} / h$ is the quantum conductance) around the Fermi level $\left(E_{f}\right)$ for both spin states. Thus, the $I-V$ curves are linear and display metallic behaviors in Figures 2(a) and 2(c). For 6ZGeNR, the transmission spectra of spin-up and spin-down states are degenerate at zero bias, and a transmission gap (TG) appears around $E_{f}$ in central panel of Figure 4(b). And there is a large continuous transmission platform when energy is beyond the TG.

As we know, the transmission spectrum can be driven by spin matching of the left and right electrodes in a spin transport device [31]. Transmission is allowed only when both the spin and orbital symmetry of the energy bands on the two electrodes are matched; otherwise, it would be forbidden. To understand the origin of the different transmission behaviors around FL in 5-ZGeNR and 6-ZGeNR devices, as shown in Figures 4(c) and 4(d), we give the isosurface plots of the wave functions of $\pi$ and $\pi^{*}$ subbands for spin-up states of 5ZGeNR and 6-ZGeNR at the $\Gamma$-point, respectively. It is known that ZGeNRs have no definite parity with respect to the $\sigma$ operation because of the buckled structure. From Figure 4(c), we cannot find any definite parity for the wave functions of $\pi$ and $\pi^{*}$ subbands in $5-Z G e N R$. However, it is noted that the center axis of 6-ZGeNR (dashed line in Figure 4(d)) is a twofold axis, and 6-ZGeNR has $c_{2}$ symmetry with respect to this axis. From Figure 4(d), we can find that the orbitals of the $\pi^{*}$ and $\pi$ subbands have odd and even parity, respectively. Therefore, the TG is equal to the energy area with the parity mismatching of the orbitals with respect to the $c_{2}$ operation. Thus, although the $\sigma$ plane is absent in ZGeNR, symmetry still plays an important role in the transport properties.

To understand the nature of the observed dual spin-filter effect, we plot the band structures of left and right electrodes and transmission spectra for $6-\mathrm{ZGeNR}$ at bias of $\pm 0.5 \mathrm{~V}$, as 


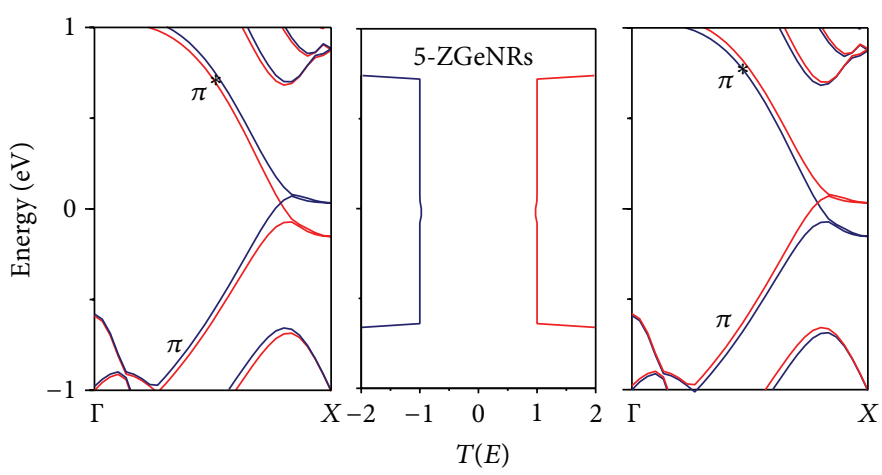

(a)

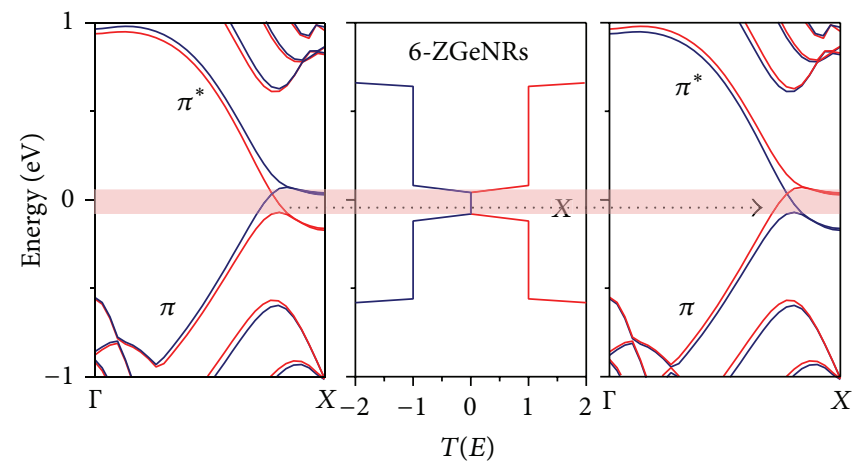

(b)
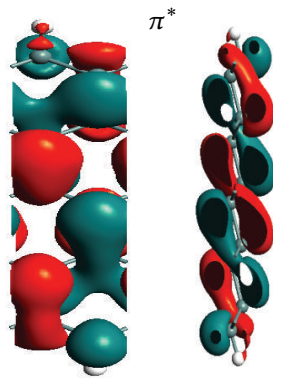

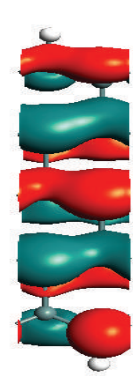

(c)
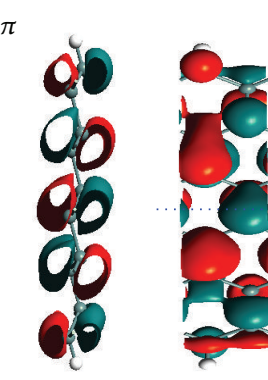

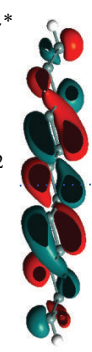

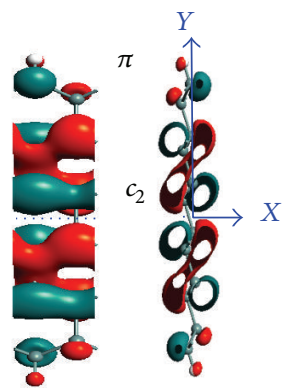

(d)

Figure 4: (a, b) Band structure for the left electrode (left panels), transmission curve (middle panels), and band structure for the right electrode (right panels) with AP configuration for 5-ZGeNR and 6-ZGeNR at zero bias, respectively. The Fermi level is set at zero. (c, d) Isosurface plots of wave functions of $\pi$ and $\pi^{*}$ subbands for spin-up states of 5-ZGeNR and 6-ZGeNR at the $\Gamma$-point, respectively.

shown in Figures 5(a) and 5(b). When the positive bias is applied, the energy bands are shifted downward (upward) for the left (right) electrode. Within the bias window, for 6-ZGeNR, as shown in Figure 5(a), the $\pi^{*}$ subband of left electrode overlaps with the $\pi$ subband of right electrode for spin-down state, since the $\pi$ and $\pi^{*}$ subbands have opposite parities (as in Figure 4(d)) with respect to the midplane mirror operation, the electron transmission from the $\pi$ subband of left electrode to the $\pi^{*}$ subband of right electrode is forbidden, and no electron transmission contributes to the current in Figure 2(g). Nevertheless, for the spin-up state, there is a wide spin-up transmission peak entering into the bias windows, which mainly originates from the coupling between the $\pi\left(\pi^{*}\right)$ subband of left electrode and the $\pi\left(\pi^{*}\right)$ subband of right electrode; thus a large spin-up current can be observed in Figure 2(e). When bias is $0.5 \mathrm{~V}$, the shifting of energy bands of the left and right electrodes and transmission matching of different spin states are just opposite to the case of $-0.5 \mathrm{~V}$, which leads to zero current for spin-up and larger current for spin-down states. As a result, the dual spin-filter effect can be observed on the $I-V$ curves of 6-ZGeNR device.

Moreover, in order to further understand the transport properties, we also show the transmission pathway at $0.24 \mathrm{eV}$ for the spin-up and spin-down currents of 6-ZGeNR under the bias of $\pm 0.5 \mathrm{~V}$. The transmission pathways $T_{i j}$ can show us the local bond contributions to the transmission coefficient. The pathways $T_{i j}$ have the property that if the system is divided into 2 parts $(A, B)$, then the pathways across the boundary between $A$ and $B$ sum up to the total transmission coefficient $T(E)=\sum_{i \in A, j \in B} T_{i j}(E)$. From Figures 5(c) and 

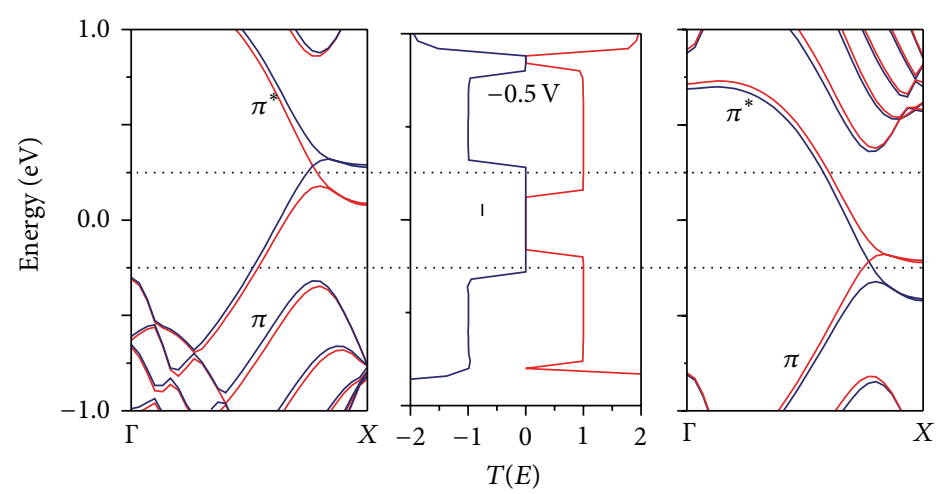

(a)
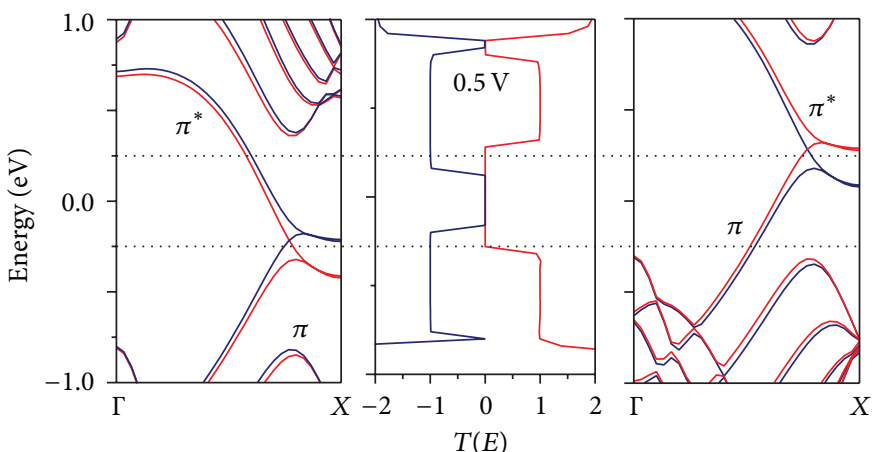

(b)

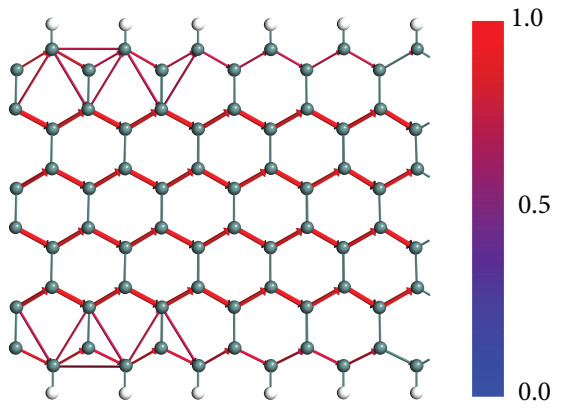

(c)

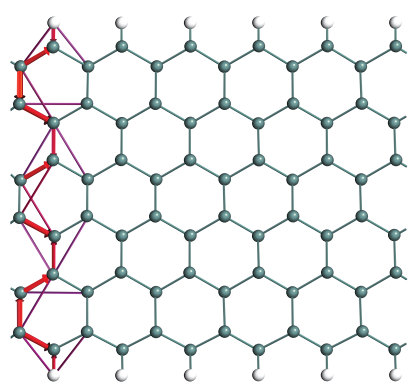

(e)

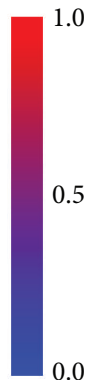

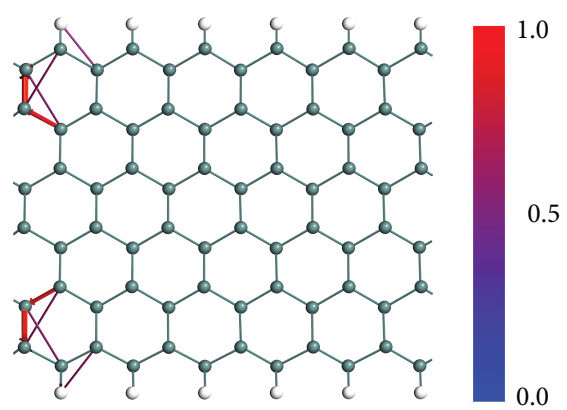

(d)

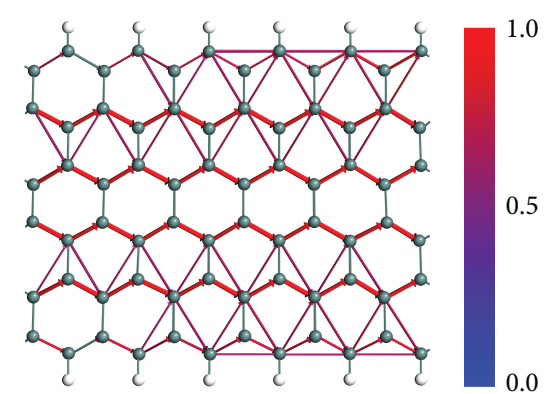

(f)

FIGURE 5: Band structure for the left electrode, transmission spectrum, and band structure for the right electrode for model 6-ZGeNR in AP configuration: (a) and (b) are for 0.5 and $-0.5 \mathrm{~V}$, respectively. (c), (d), (e), and (f) refer to the transmission pathways at $0.24 \mathrm{eV}$ for spin-up state at $-0.5 \mathrm{~V}$, spin-down state at $-0.5 \mathrm{~V}$, spin-up state at $0.5 \mathrm{~V}$, and spin-down state at $0.5 \mathrm{~V}$, respectively. The horizontal dotted lines indicate the chemical potentials of the left and right electrodes. The Fermi level is set to zero. 


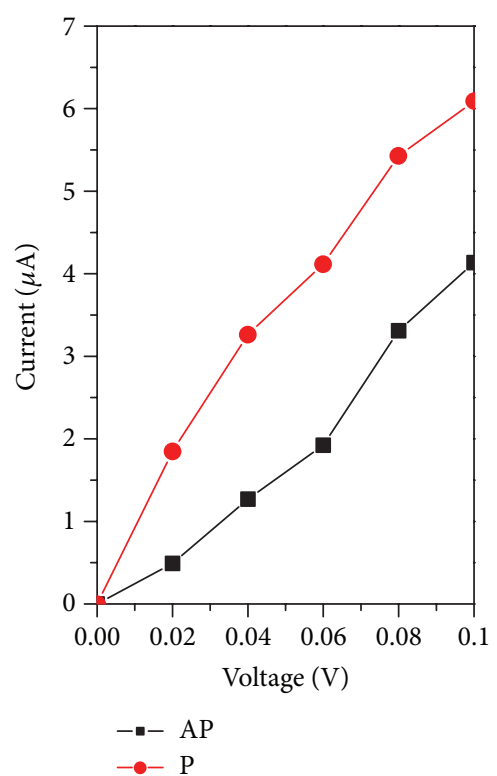

(a)

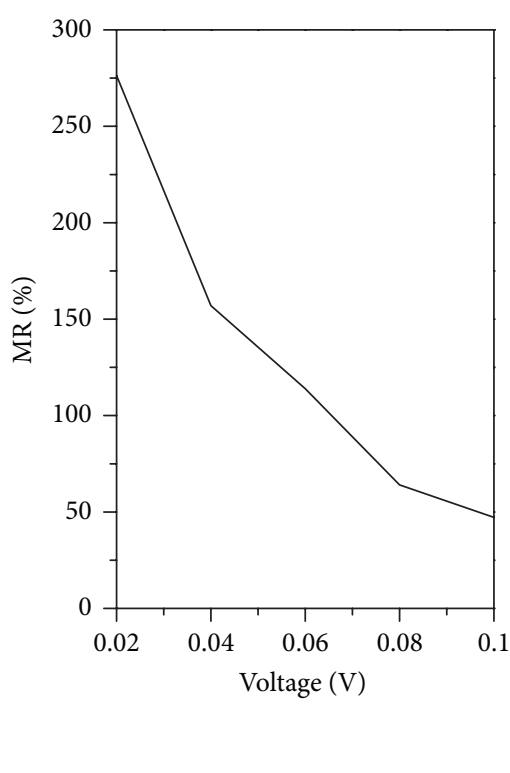

(b)

FIgure 6: (a) The $I-V$ curves for 6-ZGeNR in P configuration and AP configuration; (b) the magnetoresistance ratio (MR) for 6-ZGeNR.

5(d), it is shown that the spin-up electrons can transfer through the whole scattering region at $-0.5 \mathrm{~V}$ and reach the other electrode, corresponding to a high transmission peak in middle panel of Figure 5(b), while for the spindown electrons, the transmission channel is forbidden, and electrons cannot reach the right electrode, corresponding to the transmission coefficient which is zero at $0.24 \mathrm{eV}$ in middle panel of Figure $5(\mathrm{~b})$. At $0.5 \mathrm{~V}$, the transmission pathways demonstrate that the current channels for the spin-up state are blocked. In contrast, the current channels of the spin-down states are unblocked, and electrons can transfer through the whole scattering region. Interestingly, we can clearly find in the GeNR-based devices that there are also two different current channels like GNR-based devices: bond current (current via a chemical bond) and hopping current (current through hopping between atoms of the same symmetry sublattice) $[12,32]$. From Figures 5(c)-5(f), we can clearly find that the hopping current is obviously weaker than the bond one under the same bias voltage.

By modulating the direction of spin polarization orientations of the left and right electrodes, giant magnetoresistance has been found in the ZGNRs and ZSiNRs [8, 21]. Thus, we wonder whether the same magnetoresistance behavior would also appear in ZGeNRs. Figure 6(a) shows the $I-V$ curves for 6-ZGeNR in P and AP spin configurations, and Figure 6(b) presents the corresponding magnetoresistance ratio (MR) as a function of applied bias. We can see that the current of 6ZGeNR in $\mathrm{P}$ configuration is much bigger than that in AP configuration at low bias voltages, and the magnetoresistance behavior appears. The magnetoresistance ratio at a certain bias is defined by $\mathrm{MR}=\left(I_{\mathrm{P}}-I_{\mathrm{AP}}\right) / I_{\mathrm{AP}}$, where $I_{\mathrm{P}}$ and $I_{\mathrm{AP}}$ are the currents in the $\mathrm{P}$ and $\mathrm{AP}$ configurations related to the same bias voltage, respectively. As shown in Figure 6(b), MR is gained at the bias voltage range from 0.01 to $0.1 \mathrm{~V}$ and its maximum reaches about $270 \%$.

Moreover, since the width effect is always important in nanoribbons [33-35], we also considered the dependence of the transport property on the width of the ZGNRs. In Figure 7(a), we show the $I-V$ curves for $N$-ZGeNRs $(N=5,7$, and 9). As can be seen, the current for odd ZGeNRs with AP spin configuration is almost independent of spin and linearly increases under low $V_{\text {bias }}$, leading to $I_{\text {up }} / I_{\text {dn }}$ of nearly one. But for even $N$-ZGeNRs $(N=6,8$, and 10), shown in Figure 7(b), the current-voltage $(I-V)$ characteristics depend on interestingly not only spin, but also the direction of $V_{\text {bias }}$, and the upspin electron substantially flows only in the negative regime of $V_{\text {bias }}$, while the down-spin electron flows only in the positive regime. Thus, it can be concluded that the width of ZGeNRs does not affect the qualitative transport properties and the dual spin-filter effects can also be observed in all widths of even ZGeNRs. The major reason is that the electronic transport properties for ZGeNRs are mainly determined by the parity of the $\pi$ and $\pi^{*}$ subbands of left and right electrodes; the parity of ZGeNRs is always maintained even when the width of the nanoribbon is changed. Meanwhile, it is interesting to note that both the spin polarization and rectifying ratio are increased with the increasing of the width as shown in Figures 7(c) and 7(d). For 10-ZGeNR, the spin splitting effect can reach $93 \%$, and rectifying ratio is 23 . This means that the performance of the GeNRs-based spin filter and spin rectifier would be modulated by controlling the ribbon width.

\section{Summary}

In conclusion, we have investigated the electronic transport properties of 5-ZGeNR and 6-ZGeNRs devices. Our results show that the symmetry-dependent transport characteristics 


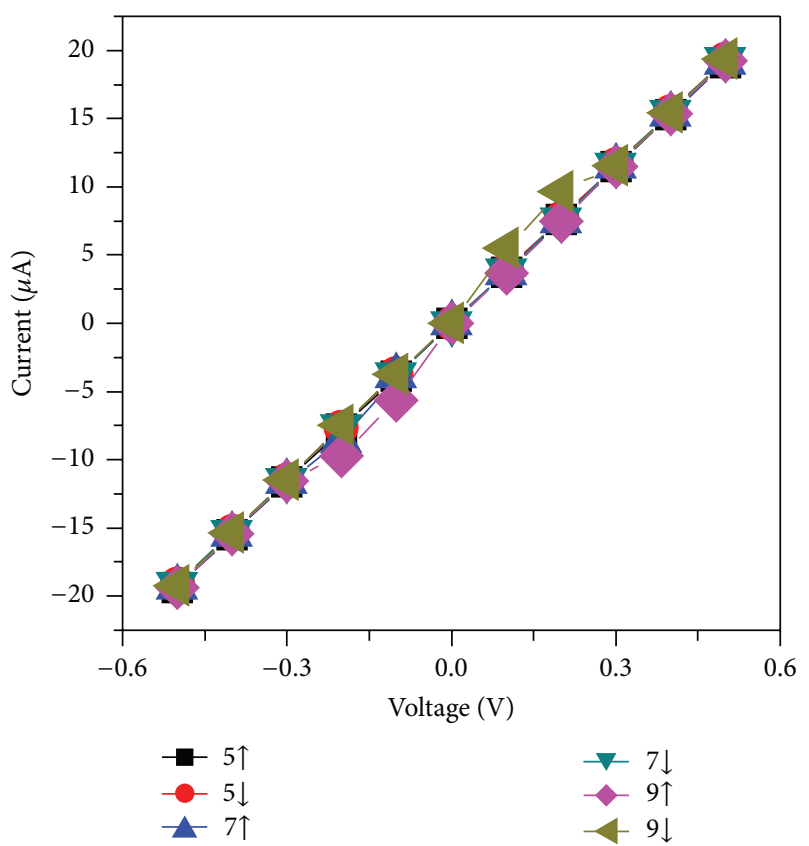

(a)

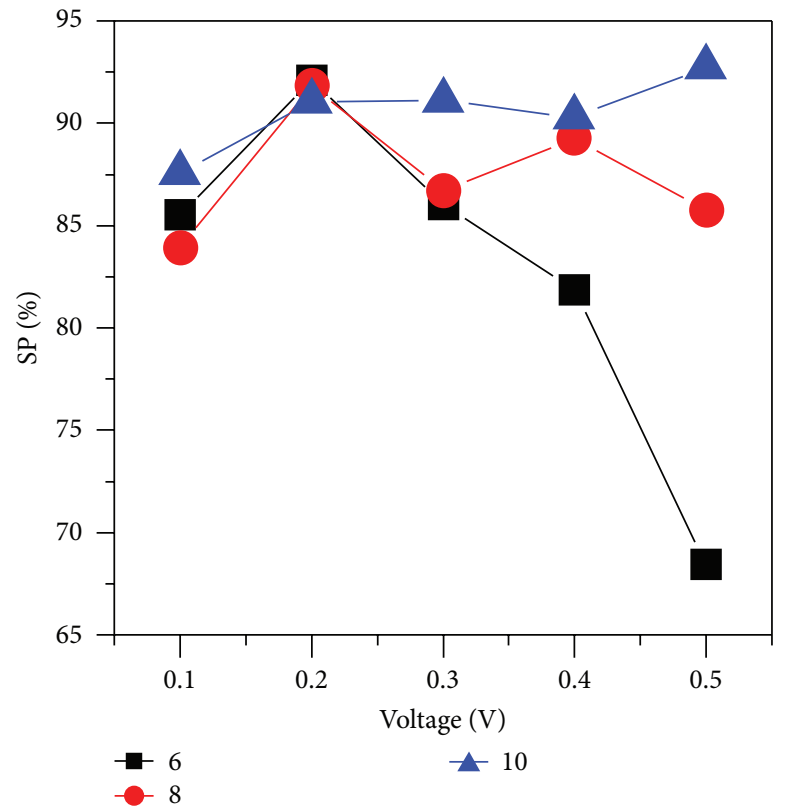

(c)

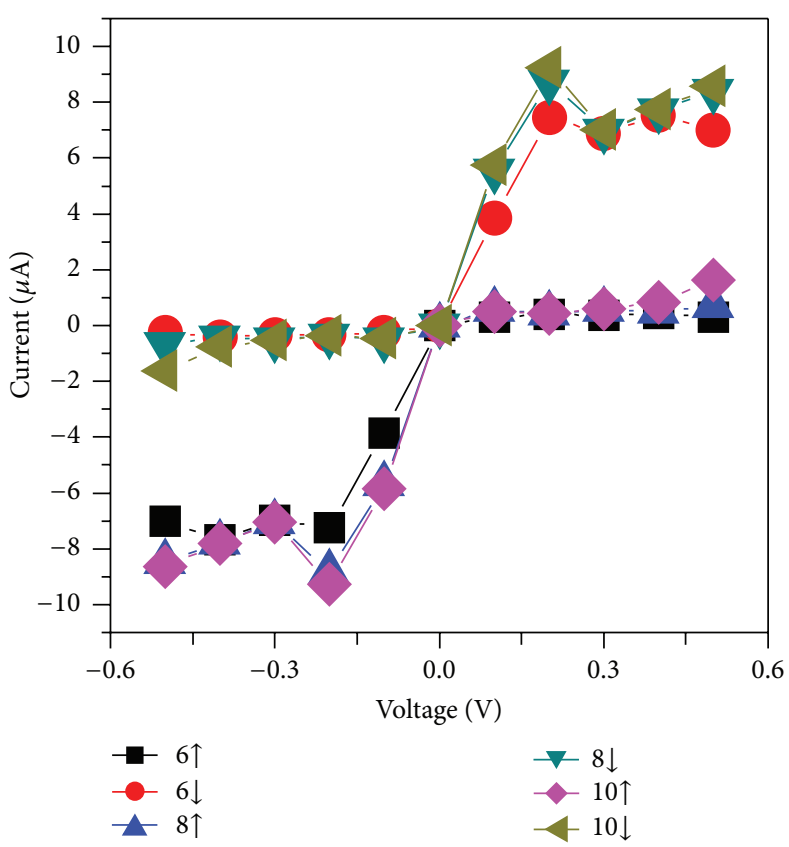

(b)

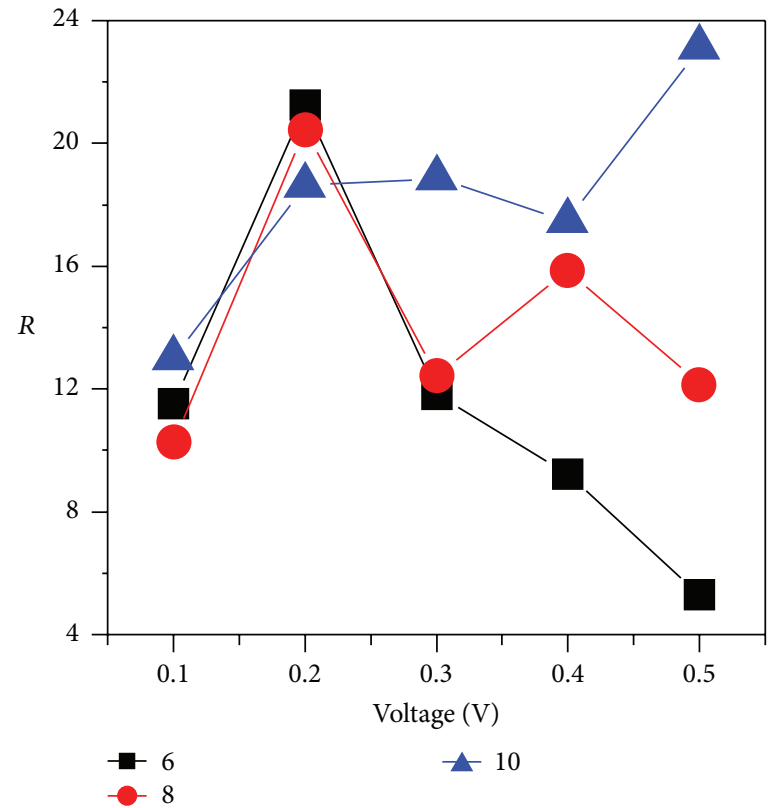

(d)

FIgURE 7: (a) $I-V$ curves for the odd ZGeNRs with different widths in AP configuration. (b) $I$ - $V$ curves for the even ZGeNRs with different widths in AP configuration. (c) Spin polarization (SP) of the even ZGeNRs. (d) Rectifying ratio of spin-up current of the even ZGeNRs with the increase of applied bias.

are observed in ZGeNRs, although the $\sigma$ mirror plane is absent, and the different symmetry of the wave functions of $\pi$ and $\pi^{*}$ subbands at the $\Gamma$-point would determine the spin-dependent transport behavior of ZGeNRs. We also find that the even 6-ZGeNR shows a dual spin-filter effect in AP magnetism configuration, but the odd 5-ZGeNR behaves as conventional conductors with linear current-voltage dependence. Moreover, it is found that when the two electrodes are in $\mathrm{P}$ configuration, the 6-ZGeNR system is in a low resistance state, while it can switch to a much higher resistance when the electrodes are in AP configuration. And the order of the corresponding magnetoresistance ratio can reach $270 \%$. We also find that the spin polarization and rectifying ratio are increased with the increasing of width in even ZGeNRs. These results may be useful in designing of high performance spin filter and spin rectifier based on GeNRs. 


\section{Conflict of Interests}

The authors declare that there is no conflict of interests regarding the publication of this paper.

\section{Acknowledgments}

This work is supported by the National Natural Science Foundation of China (Grants nos. 21103232 and 61306149), the Natural Science Foundation of Hunan Province (no. 14JJ3026), China Postdoctoral Science Foundation (nos. 2013M542130 and 2014M552145), Hong Kong Scholars Program (no. XJ2013003), and Shenghua Lieying Scholarship by the Central South University.

\section{References}

[1] X. L. Li, X. R. Wang, L. Zhang, S. W. Lee, and H. J. Dai, "Chemically derived, ultrasmooth graphene nanoribbon semiconductors," Science, vol. 319, no. 5867, pp. 1229-1232, 2008.

[2] H. Xiang, E. Kan, S.-H. Wei, M.-H. Whangbo, and J. Yang, “'Narrow' graphene nanoribbons made easier by partial hydrogenation," Nano Letters, vol. 9, no. 12, pp. 4025-4030, 2009.

[3] K. A. Ritter and J. W. Lyding, "The influence of edge structure on the electronic properties of graphene quantum dots and nanoribbons," Nature Materials, vol. 8, no. 3, pp. 235-242, 2009.

[4] S. Li, C. K. Gan, Y.-W. Son, Y. P. Feng, and S. Y. Quek, "Anomalous length-independent frontier resonant transmission peaks in armchair graphene nanoribbon molecular wires," Carbon, vol. 76, pp. 285-291, 2014.

[5] J. M. Cai, C. A. Pignedoli, L. Talirz et al., "Graphene nanoribbon heterojunctions," Nature Nanotechnology, vol. 9, no. 11, pp. 896900, 2014.

[6] X.-J. Zhang, K.-Q. Chen, L.-M. Tang, and M.-Q. Long, "Electronic transport properties on V-shaped-notched zigzag graphene nanoribbons junctions," Physics Letters A, vol. 375, no. 37, pp. 3319-3324, 2011.

[7] A. Saraiva-Souza, M. Smeu, H. Terrones, A. G. S. Filho, and M. A. Ratner, "Spin transport of polyacetylene chains bridging zigzag graphene nanoribbon electrodes: a nonequilibrium treatment of structural control and spin filtering," Journal of Physical Chemistry C, vol. 117, no. 41, pp. 21178-21185, 2013.

[8] P. Zhao, Q. H. Wu, H. Y. Liu, D. S. Liu, and G. Chen, “A first-principles study of the spin transport properties of a $4 \mathrm{H}$ TAHDI-based multifunctional spintronic device with graphene nanoribbon electrodes," Journal of Materials Chemistry C, vol. 2, no. 32, pp. 6648-6654, 2014.

[9] E.-J. Kan, Z. Li, J. Yang, and J. G. Hou, "Half-metallicity in edge-modified zigzag graphene nanoribbons," Journal of the American Chemical Society, vol. 130, no. 13, pp. 4224-4225, 2008.

[10] W. Y. Kim and K. S. Kim, "Prediction of very large values of magnetoresistance in a graphene nanoribbon device," Nature Nanotechnology, vol. 3, no. 7, pp. 408-412, 2008.

[11] P. Zhao, Q. H. Wu, D. S. Liu, and G. Chen, "Spin-filtering, giant magnetoresistance, rectifying and negative differential resistance effects in planar four-coordinate Fe complex with graphene nanoribbon electrodes," Journal of Chemical Physics, vol. 140, no. 4, Article ID 044311, 5 pages, 2014.

[12] X. Q. Deng, Z. H. Zhang, G. P. Tang, Z. Q. Fan, and C. H. Yang, "Spin filter effects in zigzag-edge graphene nanoribbons with symmetric and asymmetric edge hydrogenations," Carbon, vol. 66, pp. 646-653, 2014.

[13] S. Cahangirov, M. Topsakal, E. Aktürk, H. Şahin, and S. Ciraci, "Two- and one-dimensional honeycomb structures of silicon and germanium," Physical Review Letters, vol. 102, no. 23, Article ID 236804, 4 pages, 2009.

[14] Q. Pang, Y. Zhang, J.-M. Zhang, and K.-W. Xu, "Functionalization of low-dimensional honeycomb germanium with 3D transition-metal atoms," Computational Materials Science, vol. 50, no. 5, pp. 1717-1724, 2011.

[15] W.-Q. Han, L. Wu, Y. Zhu, and M. Strongin, "In-situ formation of ultrathin Ge nanobelts bonded with nanotubes," Nano Letters, vol. 5, no. 7, pp. 1419-1422, 2005.

[16] Q. Pang, Y. Zhang, J.-M. Zhang, V. Ji, and K.-W. Xu, "Electronic and magnetic properties of perfect and defected germanium nanoribbons," Materials Chemistry and Physics, vol. 130, no. 12, pp. 140-146, 2011.

[17] H.-Z. Lu, W. Yao, D. Xiao, and S.-Q. Shen, "Intervalley scattering and localization behaviors of spin-valley coupled dirac fermions," Physical Review Letters, vol. 110, no. 1, Article ID 016806, 5 pages, 2013.

[18] H. Pan, Z. Li, C.-C. Liu, G. Zhu, Z. Qiao, and Y. Yao, "Valleypolarized quantum anomalous hall effect in silicene," Physical Review Letters, vol. 112, no. 10, Article ID 106802, 5 pages, 2014.

[19] F. Liu, C.-C. Liu, K. Wu, F. Yang, and Y. Yao, “ $d+i d^{\prime}$ chiral superconductivity in bilayer silicene," Physical Review Letters, vol. 111, no. 6, Article ID 066804, 5 pages, 2013.

[20] P. De Padova, C. Quaresima, C. Ottaviani et al., "Evidence of graphene-like electronic signature in silicene nanoribbons," Applied Physics Letters, vol. 96, Article ID 261905, 3 pages, 2010.

[21] J. Kang, F. Wu, and J. B. Li, "Symmetry-dependent transport properties and magnetoresistance in zigzag silicene nanoribbons," Applied Physics Letters, vol. 100, no. 23, Article ID 233122, 2012.

[22] T. P. Kaloni and U. Schwingenschlögl, "Weak interaction between germanene and $\mathrm{GaAs}(0001)$ by $\mathrm{H}$ intercalation: a route to exfoliation," Journal of Applied Physics, vol. 114, no. 18, Article ID 184307, 4 pages, 2013.

[23] M. Ezawa, "Quantized conductance and field-effect topological quantum transistor in silicene nanoribbons," Applied Physics Letters, vol. 102, no. 17, Article ID 172103, 5 pages, 2013.

[24] X.-S. Ye, Z.-G. Shao, H. Zhao, L. Yang, and C.-L. Wang, "Intrinsic carrier mobility of germanene is larger than graphene's: firstprinciple calculations," RSC Advances, vol. 4, no. 41, pp. 2121621220, 2014.

[25] E. J. Loren, J. Rioux, C. Lange, J. E. Sipe, H. M. van Driel, and A. L. Smirl, "Hole spin relaxation and intervalley electron scattering in germanium," Physical Review B, vol. 84, no. 21, Article ID 214307, 2011.

[26] A. Jain, C. Vergnaud, J. Peiro et al., "Electrical and thermal spin accumulation in germanium," Applied Physics Letters, vol. 101, no. 2, Article ID 022402, 4 pages, 2012.

[27] Z. Y. Li, H. Y. Qian, J. Wu, B. L. Gu, and W. H. Duan, "Role of symmetry in the transport properties of graphene nanoribbons under bias," Physical Review Letters, vol. 100, no. 20, Article ID 206802, 4 pages, 2008.

[28] Q. Yue, S. L. Chang, J. C. Tan, S. Q. Qin, J. Kang, and J. Li, "Symmetry-dependent transport properties and bipolar spin filtering in zigzag $\alpha$-graphyne nanoribbons," Physical Review B, vol. 86, no. 23, Article ID 235448, 8 pages, 2012. 
[29] M. Brandbyge, J. L. Mozos, P. Ordejón, J. Taylor, and K. Stokbro, "Density-functional method for nonequilibrium electron transport," Physical Review B, vol. 65, no. 16, Article ID 165401, 3 pages, 2002.

[30] J. Taylor, H. Guo, and J. Wang, “Ab initio modeling of open systems: charge transfer, electron conduction, and molecular switching of a $\mathrm{C}_{60}$ device," Physical Review B, vol. 63, no. 12, Article ID 121104, 4 pages, 2001.

[31] L. Cui, B. Yang, X. Li, C. Cao, and M. Q. Long, "Effects of symmetry and spin configuration on spin-dependent transport properties of iron-phthalocyanine-based devices," Journal of Applied Physics, vol. 116, Article ID 033701, 4 pages, 2014.

[32] A. H. C. Neto, F. Guinea, N. M. R. Peres, K. S. Novoselov, and A. K. Geim, "The electronic properties of graphene," Reviews of Modern Physics, vol. 81, article 109, 5 pages, 2009.

[33] M.-Q. Long, L. Tang, D. Wang, L. J. Wang, and Z. G. Shuai, "Theoretical predictions of size-dependent carrier mobility and polarity in graphene," Journal of the American Chemical Society, vol. 131, no. 49, pp. 17728-17729, 2009.

[34] Y. Y. Liang, V. Wang, H. Mizuseki, and Y. Kawazoe, "Band gap engineering of silicene zigzag nanoribbons with perpendicular electric fields: a theoretical study," Journal of Physics: Condensed Matter, vol. 24, no. 45, Article ID 455302, 8 pages, 2012.

[35] K. Yang, S. Cahangirov, A. Cantarero, A. Rubio, and R. D’Agosta, "Thermoelectric properties of atomically thin silicene and germanene nanostructures," Physical Review B, vol. 89, no. 12, Article ID 125403, 13 pages, 2014. 

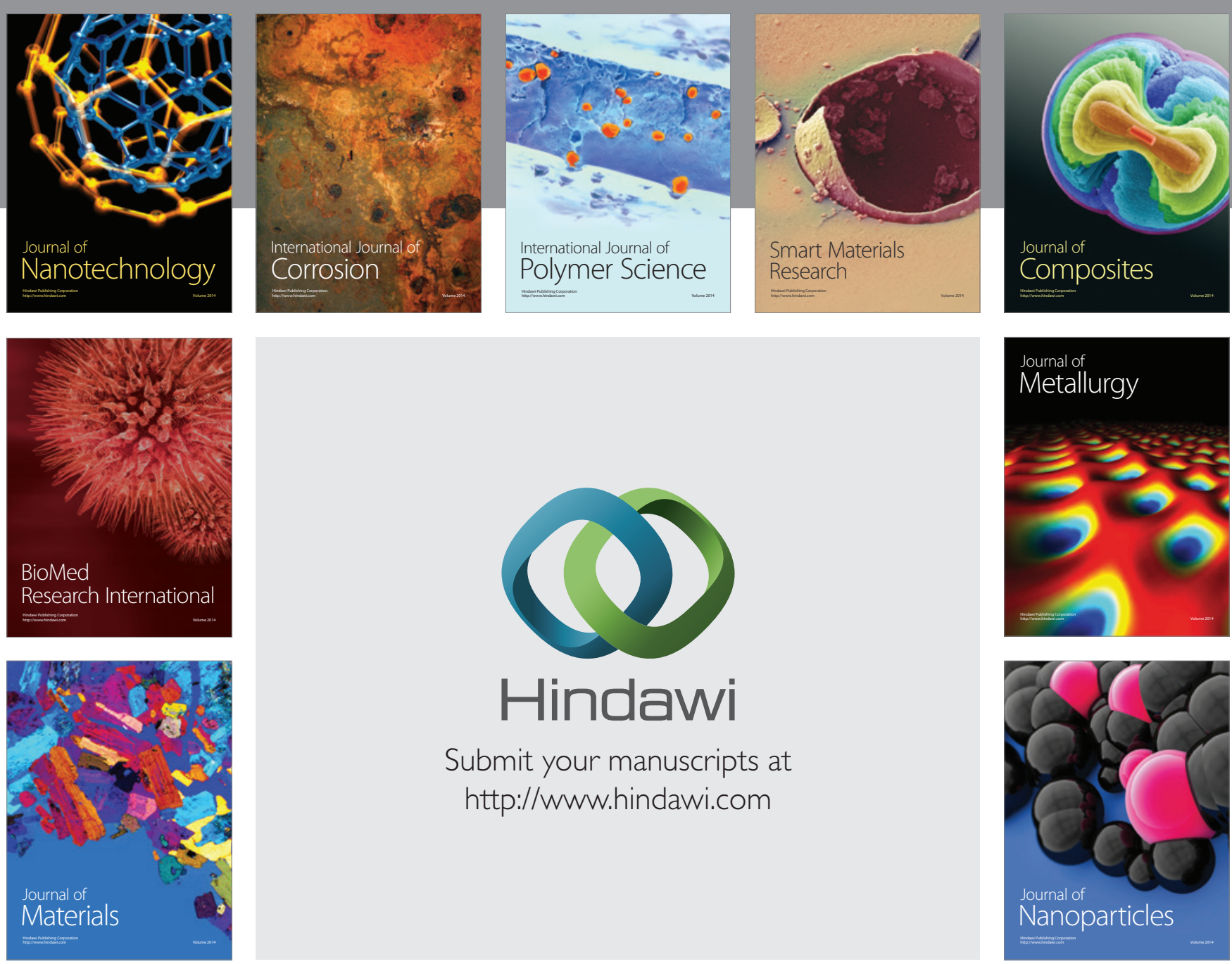

Submit your manuscripts at http://www.hindawi.com
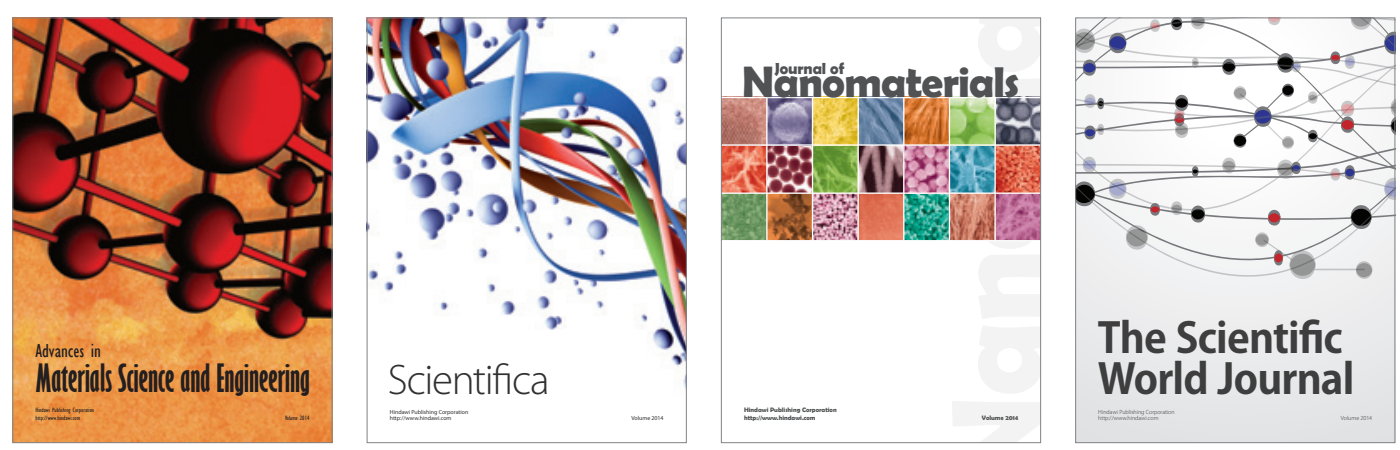

\section{The Scientific World Journal}
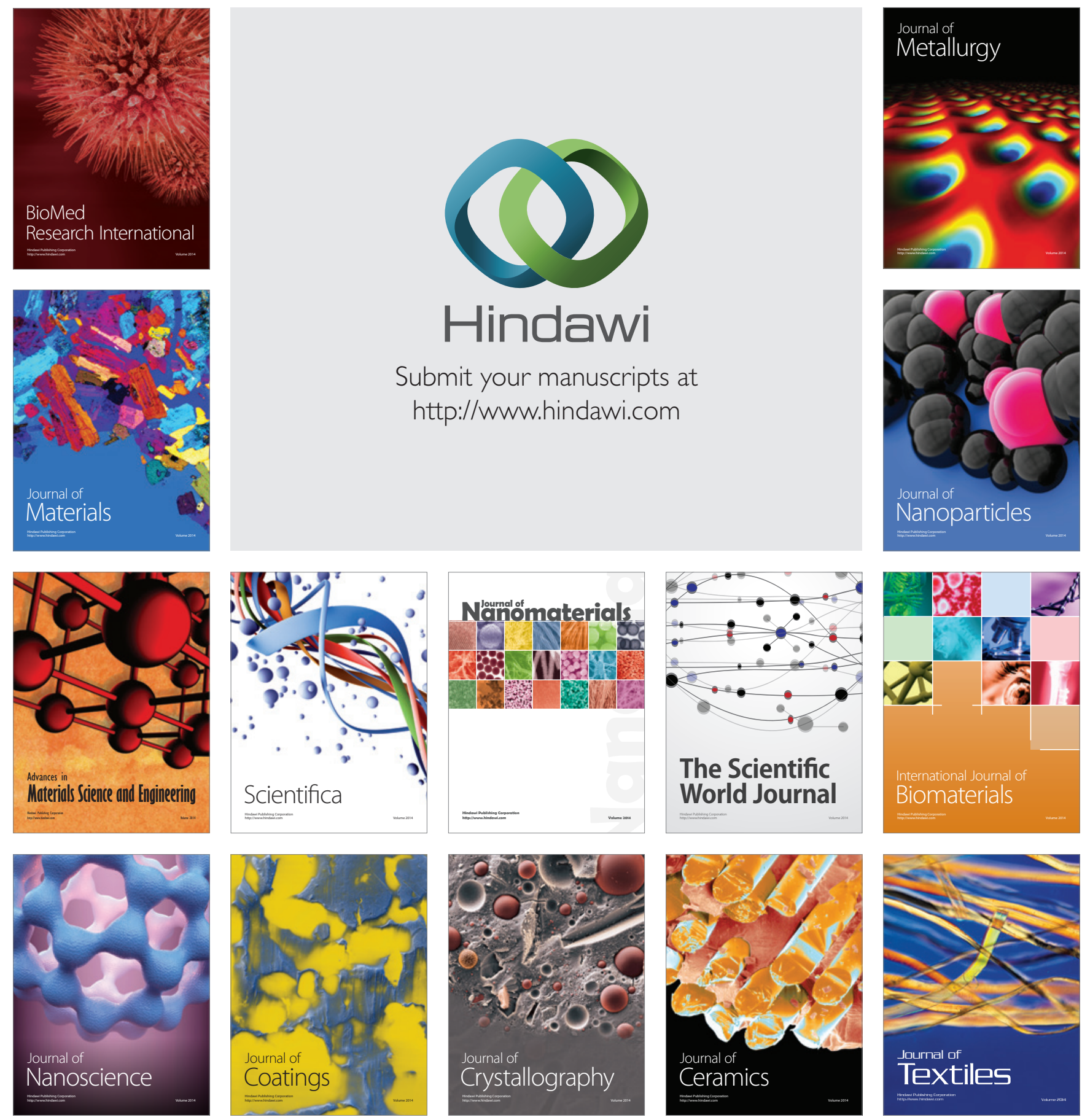